\title{
Topiramate-induced Acute Transient Myopia and Angle Narrowing
}

\author{
Summaya Khan, Ammarah Ashraf, Hassaan Javaid and Muhammad Azeem Khizer \\ Armed Forces Institute of Ophthalmology, Rawalpindi, Pakistan
}

\begin{abstract}
Sulphamate drugs, widely prescribed for various systemic conditions, are reported to have rare ocular adverse-effects, usually within weeks of initiation of treatment. Medical and drug history in such cases are of pivotal importance in reaching a proper diagnosis. This study reports three cases, which developed topiramate-induced ocular side effects. In one of the cases, although the angles were narrow in both eyes, yet intra-ocular pressure (IOP) was not high. Also, in the third case, there were no macular striae. Topiramate was immediately withheld and all cases were improved without any permanent ocular damage.
\end{abstract}

Key Words: Sulphamate, Topiramate, Angle closure glaucoma, Myopia.

How to cite this article: Khan S, Ashraf A, Javaid H, Khizer MA. Topiramate-induced Acute Transient Myopia and Angle Narrowing. J Coll Physicians Surg Pak 2021; 31(06):728-731.

\section{INTRODUCTION}

Topiramate is a sulphamate-substituted antiepileptic drug used for the treatment of seizures and as prophylaxis for several conditions including migraine, cluster headaches and bipolar affective disorder. A number of ocular adverse-effects have been described with use of topiramate including bilateral angle closure, acute myopia, choroidal effusion, nystagmus, diplopia, periorbital edema and blepharospasm. ${ }^{1,2}$ Researchers have reported cases of topiramate-induced acute myopia with angle narrowing to emphasis upon the relevance of drug history, especially when managing a patient with bilateral acute angle narrowing. ${ }^{3,4}$

\section{CASE NO. 1:}

A 24-year woman presented with episodes of headache over the last 10 days, and blurred vision in both eyes since morning. Her symptoms began 10 days back, when she had started having episodes ofsevereleft-sided headache, which wasthrobbing in nature. She had consulted a neurologist for her condition and was put on oral topiramate, $25 \mathrm{mg}$ twice a day, for two weeks. After 10 days of taking topiramate, she woke up with blurred vision and was unable to see beyond the stretch of her arms. She stated that her vision was fine prior to this episode and she never felt the need for corrective lenses. Her medical history and systemic examination were unremarkable. She had no known drug allergies previously.

Correspondence to: Dr. Ammarah Ashraf, Armed Forces Institute of Ophthalmology, Rawalpindi, Pakistan

E-mail: haim199191@gmail.com

Received: September 24, 2019; Revised: February 04, 2020; Accepted: February 12, 2020

DOI: https://doi.org/10.29271/jcpsp.2021.06.728

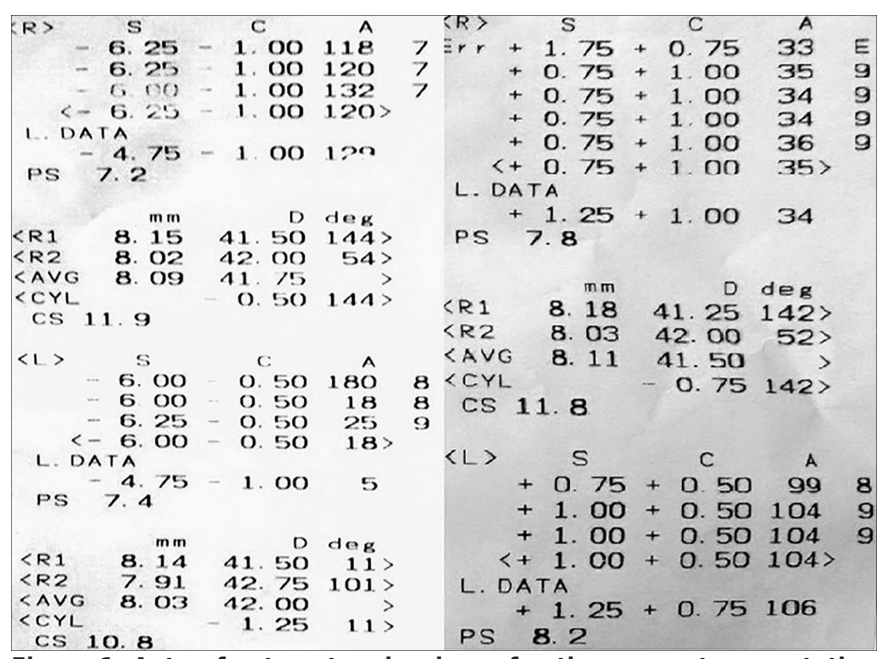

Figure 1: Autorefractometry showing refractive error at presentation (right) and on day 5 of discontinuation of topiramate (left).

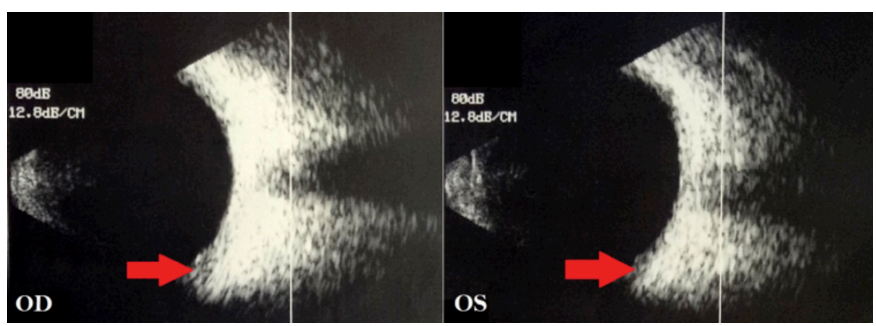

Figure 2: B-Scan showing bilateral peripheral choroidal effusion at the time of presentation.

On examination, she had a visual acuity (VA) of counting fingers at $1 \mathrm{~m}$ in each eye, not improving with refraction or pinhole. Auto refractometer (AR) showed a refractive error of -6.00 dioptres sphere in each eye (Figure 1). Slit-lamp examination showed bilateral clear cornea, shallow anterior chambers (Van Herrick 1 ) and equally reactive pupils with no signs of inflammation. Intraocular pressure (IOP) was $14 \mathrm{~mm} \mathrm{Hg}$ in right as well as left 
eye as measured by Goldmann Applanation tonometer. Dynamic gonioscopy performed with zeiss goniolens revealed Shaffer Grade 1 in all quadrants in both eyes with no apposition. Fundus examination revealed flat retina, healthy optic discs and chorioretinal folds at the macula in both eyes. Ultrasound Bscan showed choroidal effusion in both eyes (Figure 2). Central anterior chamber depth (ACD) measured using IOL Master 700 (Zeiss) was $2.63 \mathrm{~mm}$ in the right and $2.61 \mathrm{~mm}$ in the left eye. Anterior segment optical coherence tomography (OCT) showed temporal anterior chamber angle to be 9.9 degrees in the right and 14.1 degrees in the left eye (Figures 3 and 4).

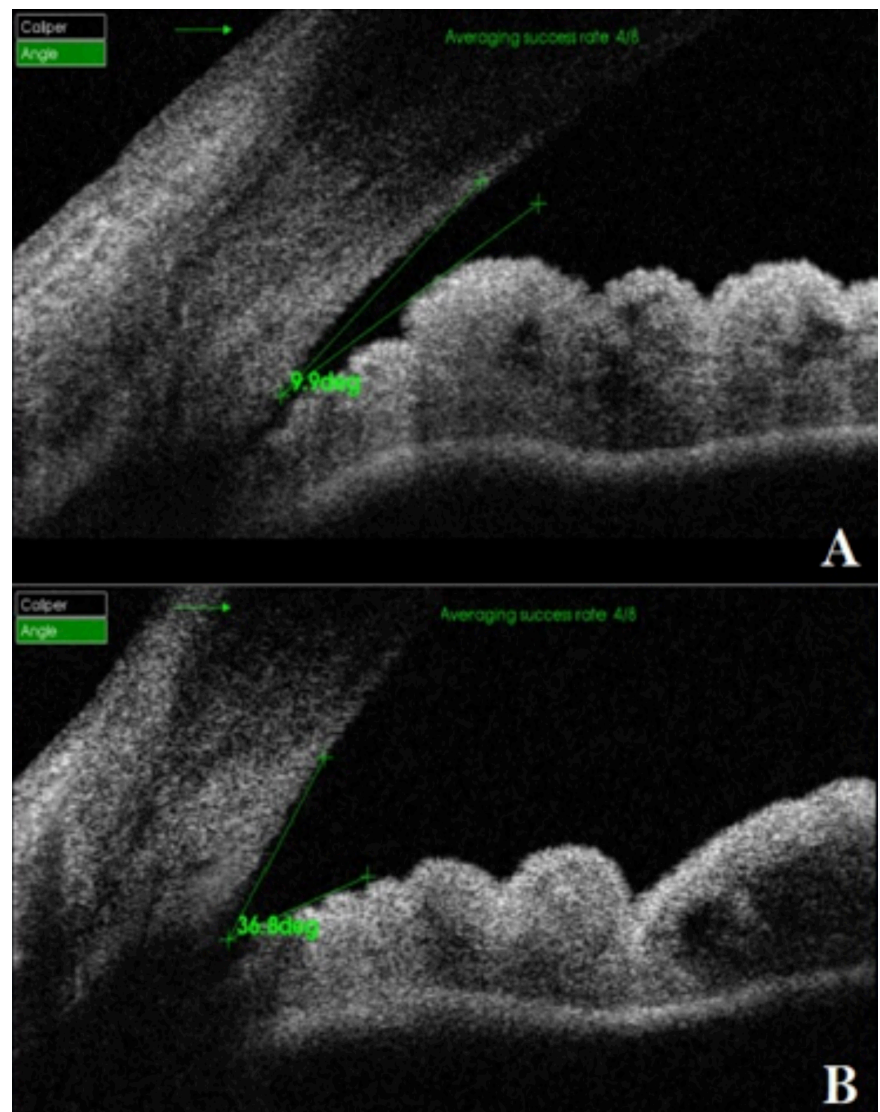

Figure 3: Anteriorsegment OCT showing AC angle of right eye at presentation (A) and after discontinuation of Topiramate (B).

A diagnosis of topiramate-induced acute myopia with angle narrowing was made. Topiramate was immediately stopped and the patient was prescribed topical $1 \%$ cyclopentolate every 8 hours along with topical 1\% prednisolone acetate every 6 hours.

On fifth day of discontinuing topiramate, AR reading had changed dramatically (Figure 1) and VA improved to 6/9 OD with +0.75 dioptre sphere correction and 6/7.5 OS with +0.50 dioptre correction. The central ACD was measured to be 3.06 $\mathrm{mm}$ in the right and $3.01 \mathrm{~mm}$ in the left eye. Anterior segment OCT was repeated which showed temporal AC angle of 36.8 degrees in the right and 33.9 degrees in the left eye (Figures 3 and 4). Over the course of one week, prescribed ocular treatment was tapered off. Patient was referred to neurologist for alternative prophylaxis of migraine and was put on regular follow-up in OPD at 2 weeks and 4 weeks showing no abnormality.

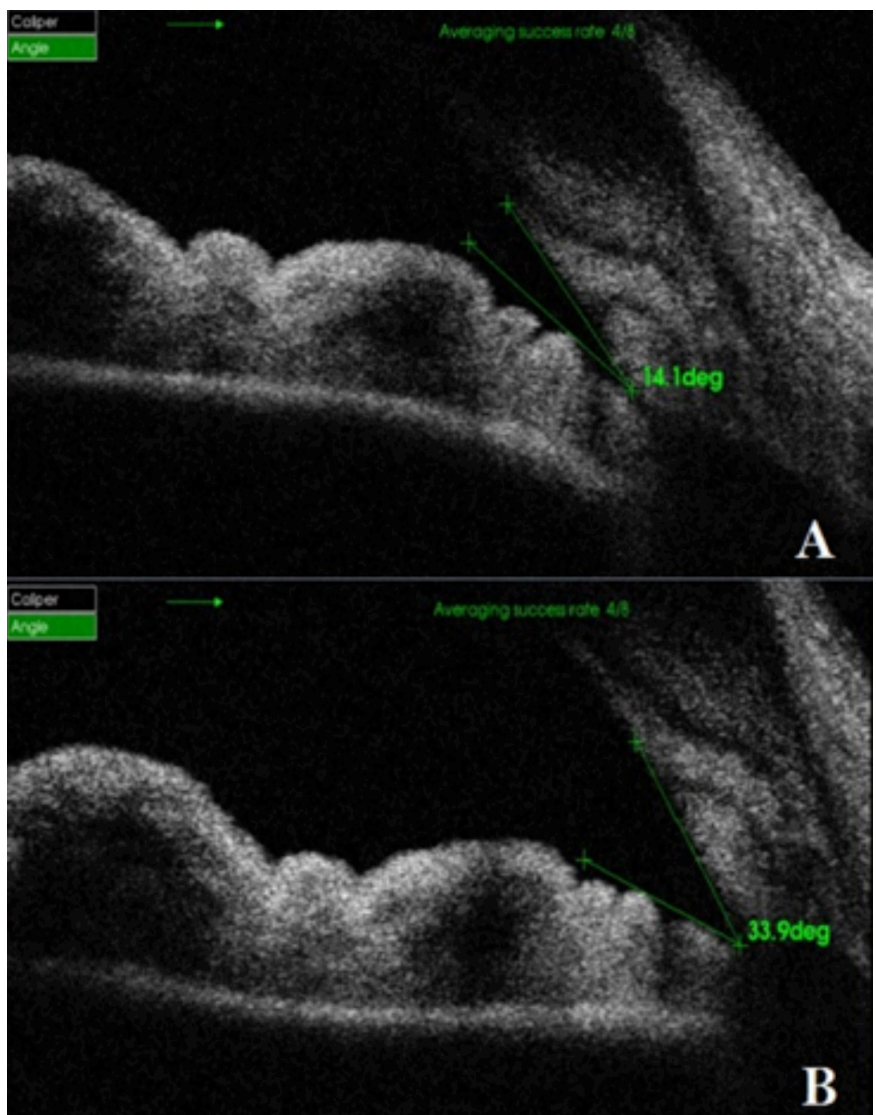

Figure 4: Interior segment OCT showing AC angle of left eye at presentation (A) and after discontinuation of Topiramate (B).

\section{CASE NO. 2:}

A26-year female reported with complain of sudden painful deterioration of vision for last one day in both eyes. On inquiring, it was revealed that she had headache for the last one week, for which she was advised oral topiramate $25 \mathrm{mg}$ by neurophysician. Past ocular and systemic history was non-contributory. Previously, no known drugallergy was elicited.

Her ocular examination revealed VA of counting finger at $2 \mathrm{~m}$ in both eyes which did not improve with refraction or pinhole. AR showed a refractive error of -6.00 and -5.75 dioptres sphere in right and left eyes, respectively (Figure 5). Slit-lamp examination showed bilateral conjunctival congestion and edematous cornea. IOP was $44 \mathrm{mmHg}$ in both eyes. Ultrasound B-scan showed bilateral choroidal effusion. Rest of the ocular examination and investigation were impossible due to bilateral edematouscornea.

Diagnosis of topiramate-induced myopic shift and acute angle closure glaucoma was made and she was treated with topical cycloplegic, steroid and IOP lowering medication. She was advised to stop taking topiramate immediately. On $2^{\text {nd }}$ day of topiramate discontinuation, her vision improved to 6/36 in right eye while 6/60 in left eye and AR reading changed to -4.00 dioptres. Her corneal edema was reduced, anterior chamber was 
shallow, pupil regularly reactive and fundi showed extensive retinal striae on macula. IOP reduced to $24 \mathrm{mmHg}$ of in right eye and $28 \mathrm{mmHg}$ in left eye. Central ACD measured with IOL Master 700 was $2.26 \mathrm{~mm}$ in the right and $2.29 \mathrm{~mm}$ in the left eye. Anterior segment OCT showed temporal anterior chamber angle to be 6.5 degrees in the right and 2.1 degrees in the left eye.

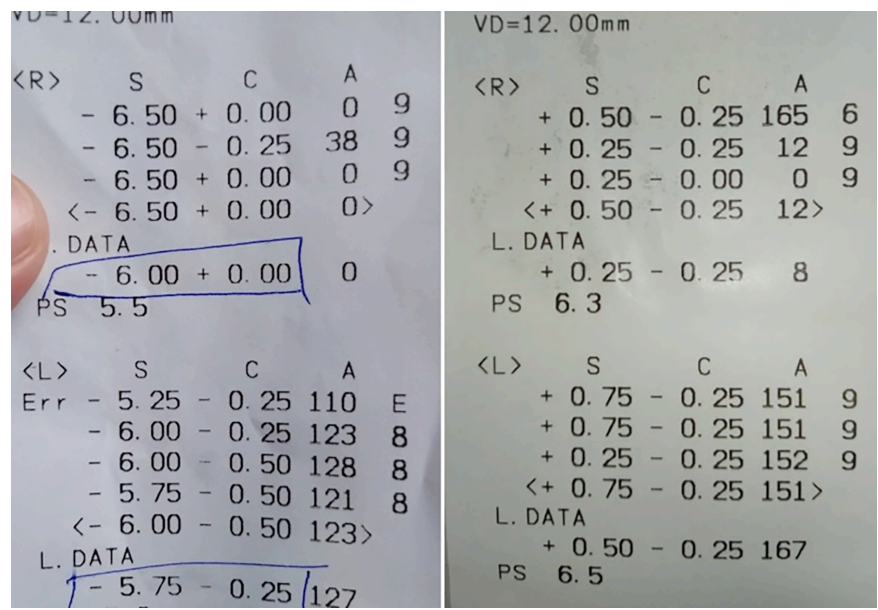

Figure 5: Auto refractometry showing refractive error at presentation (right) and after (left) completion of treatment.

After two weeks of treatment, her VA was $6 / 6$ in both eyes and AR reading was +0.25 DS/ - 0.25 DC and + 0.50 DS/ - 0.25 DC in right and left eye, respectively. Bilaterally cornea was clear with deep anterior chamber and retinal striae completely resolved. Ultrasound revealed complete resolution of choroidal effusion and anterior segment OCT showed temporal angle to be 48.4 degrees in the right eye and 52.7 degrees in left eye. Ocular treatment was stopped and patient was followed-up up to 6 weeks with no active complains.

\section{CASE NO. 3:}

A 33-year female presented with sudden deterioration of vision in both eyes for the last six hours. She was a known case of migraine and her neurophysician prescribed topiramate $25 \mathrm{mg}$ a week earlier. She was otherwise healthy with no significant systemic and ocular history. There was no history of drug allergy previously.

VA was 6/60 in both eyes, which did not improve with pinhole or refraction. AR showed a refractive error of $-4.50 \mathrm{DS} /-0.50 \mathrm{DC}$ and $-4.25 \mathrm{DS} / \mathrm{-0.50} \mathrm{DC}$ in right and left eyes, respectively (Figure 6). Slit-lamp examination showed bilateral conjunctival congestion and shallow anterior chamber (Van Herrick Grade 1). IOP measured was 28 and $26 \mathrm{mmHg}$ in right and left eyes, respectively. Contrary to aforementioned cases, there were no chorioretinal folds on macula in this case. Ultrasound B-scan showed very minimal bilateral choroidal effusion. Anterior segment OCT showed temporal anterior chamber angle to be 7.9 degrees in the right eye and 11.1 degrees in the left eye.

Topiramate was immediately discontinued and topical cyclopentolate, steroid and pressure lowering agents were prescribed. Patient was reviewed after three days. VAimproved to $6 / 6$ in both eyes and anterior chamber depth improved. IOP was $16 \mathrm{mmHg}$ in right while $14 \mathrm{mmHg}$ in left eye. AR reading was +0.50 DS/-1.00 DC in both eyes. Anterior segment OCT showed temporal anterior chamber angle to be 22.1 degrees in the right eye and 25.9 degrees in the left eye. Patient was asked to continue treatment for one week with follow-up review after two weeks and four weeks.

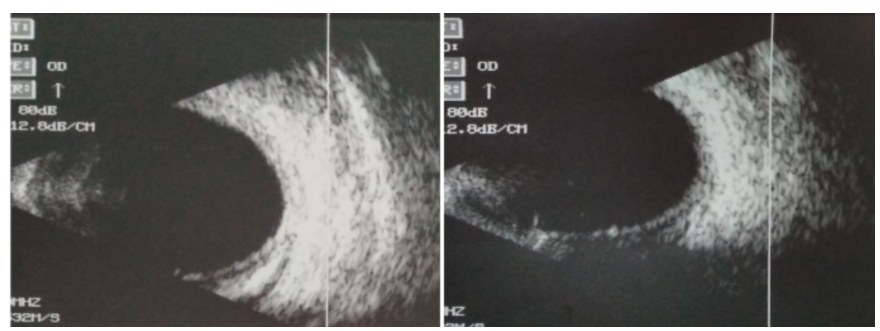

Figure 6: Auto refractometry showing refractive error at presentation (right) and on day 5 of discontinuation of topiramate (left).

\section{DISCUSSION}

Acute myopia is a rare idiosyncratic reaction to sulphonamides, and was first described in $1938 .^{2}$ Topiramate is a sulfamate prescribed as a first line prophylactic agent for migraine along with a number of other indications. Acute myopia, acute secondary angle closure glaucoma, and suprachoroidal effusion are complications of topiramate use suggested by WHO Causality Assessment. ${ }^{3}$ Although controversy exists regarding the exact mechanism of acute myopia and angle-closure glaucoma after topiramate use, most authors have attributed this to ciliochoroidal effusion with forward displacement of lens-iris diaphragm and shallowness of anterior chamber. Weak carbonic anhydrase inhibition and prostaglandin-mediated effects, exhibited by topiramate, are thought to play a role. A review of literature shows that a vast majority of patients who experienced these ocular side effects are females falling in the 20 to 39 years age group, similar to the cases in this study. Moreover, these adverse effects were seen within two weeks of initiation of treatment with topiramate in most of the cases. $^{4}$

Treatment of this condition involves discontinuation or replacement of the causative drug in consultation with the treating physician. It is important to counsel the patient regarding the disease and the reversible nature of the symptoms. Cycloplegics may help in reversing the process by causing traction on the ciliary body and decreasing lens thickness. Steroids can be prescribed to decrease ciliary effusion. Topical or systemic ocular hypotensive agents may be needed to control the IOP. However, miotics, which are the mainstay of treatment in primary angle closure glaucoma are contraindicated here, as they can further aggravate the condition by moving forward the lens-iris diaphragm. As the mechanism of angle closure does not involve pupillary block, peripheral iridectomy is not useful in the treatment of this type of secondary angle-closure glaucoma. ${ }^{5,6}$

We conclude that the prescribing physician, ophthalmologist and the patient should be aware of these rare but serious adverse effects of the drug; and seek immediate ophthalmic 
consultation, when needed. As evident from the case studies, it is highly emphasised to obtain a detailed drug history before advising topiramate. Early recognition and discontinuation of topiramate is necessary for the early recovery of the patient.

\section{PATIENTS' CONSENT:}

Informed consents were taken from all the patients before reporting these cases.

\section{CONFLICT OF INTEREST:}

The authors declared no conflict of interest.

\section{AUTHORS' CONTRIBUTION:}

SK: Final approval of manuscriptand final review.

$A A, H J$ : Data collection and drafting manuscript.

MAK: Design of the work.

\section{REFERENCES}

1. Fraunfelder FW, Fraunfelder FT, Keates EU. Topiramate- associated acute, bilateral, secondary angle-closure glaucoma. Ophthalmology 2004; 111(1):109-11. doi: 10.1016/j.ophtha.2003.04.004.

2. Berns W. Proceedings of the meeting of the Swedish ophthalmological society, 3rd December, 1938. Acta Ophthalmol 1940; 1896-98.

3. Zaki A. Adverse drug reaction and causality assessment scales, lung India, 2011; 28(2):152-3. DOI:10.4103/ 0970-2113.80343

4. Thambi L, Kapcala LP, Chambers W, Nourjah P, Beitz J, Chen $\mathrm{M}$, et al. Topiramate-associated secondary angleclosure glaucoma: A case series. Arch Ophthalmol 2002; 120(8):1108. doi: 10.1001/archopht.120.8.1108.

5. Boentert M, Aretz H, Ludemann P. Acute myopia and angleclosure glaucoma induced by topiramate. Neurology 2003; 61(9):1306. doi: 10.1212/01.wnl.0000091425.43083.c1.

6. Chen TC, Chao CW, Sorkin JA. Topiramate-induced myopic shift and angle closure glaucoma. Br J Ophthalmol 2003; 87(5):648-9. doi: 10.1136/bjo.87.5.648. 